\title{
BRANCHED COVERS OF TANGLES IN THREE-BALLS
}

\author{
MAKIKO ISHIWATA \\ Department of Mathematics, Tokyo Woman's Christian University \\ Zempukuji 2-6-1, Suginamiku, Tokyo 167-8585, Japan \\ e-mail: mako@twcu.ac.jp \\ JÓZEF H. PRZYTYCKI \\ Department of Mathematics, The George Washington University \\ Washington, DC 20052, USA \\ e-mail: przytyck@research.circ.gwu.edu \\ AKIRA YASUHARA \\ Department of Mathematics, Tokyo Gakugei University \\ Nukuikita 4-1-1, Koganei, Tokyo 184-8501, Japan \\ e-mail: yasuhara@u-gakugei.ac.jp
}

\begin{abstract}
We give an algorithm for a surgery description of a $p$-fold cyclic branched cover of $B^{3}$ branched along a tangle. We generalize constructions of Montesinos and Akbulut-Kirby.
\end{abstract}

2000 Mathematics Subject Classification. Primary 57M25; Secondary 57M12

Key Words and Phrases. tangle, branched cover, surgery, Heegaard decomposition

Tangles were first studied by Conway ⿶凵 prime and hyperblic knots. A branched cover of a tangle is an indispensable tool for understanding tangles. Hence it is important to give practical presentations of branched covers of tangles. A $p$-fold branched cover of an $n$-tangle is a three-manifold with the boundary a connected surface of genus $(n-1)(p-1)$. Such a manifold can be obtained from the genus $(n-1)(p-1)$ handlebody by a surgery. We provide an algorithm for a surgery description of a $p$-fold cyclic branched cover of $B^{3}$ branched along a tangle. The 
construction generalizes that of Montesinos [7] and Akbulut and Kirby [1]. It is strikingly simple in the case of the two-fold branched cover. We also discuss the related Heegaard decomposition of a $p$-fold branched cover of an $n$-tangle.

\section{Sugery descriptions}

A tangle is a one-manifold properly embedded in a three-ball. An $n$-tangle is a tangle with $2 n$ boundary points. Let $T$ be an $n$-tangle and $T_{0}$ a trivial $n$-tangle diagram円 (Fig. 1). Let $D_{1} \cup \cdots \cup D_{n}$ be a disjoint union of disks bounded by $T_{0}$ and $b_{1}, \cdots, b_{m}$ be mutually disjoint disks in $B^{3}$ such that $b_{i} \cap \bigcup_{j} D_{j}=\partial b_{i} \cap T_{0}$ are two disjoint arcs in $\partial b_{i}(i=1, \cdots, m)$, see Fig. 2. We denote by $\Omega\left(T_{0} ;\left\{D_{1}, \cdots, D_{n}\right\},\left\{b_{1}, \cdots, b_{m}\right\}\right)$ the tangle $T_{0} \cup \bigcup_{i} \partial b_{i}-\operatorname{int}\left(T_{0} \cap \bigcup_{i} \partial b_{i}\right)$ and call it a disk-band representation 2 of a tangle. A disk-band representation is called bicollared if the surface $\bigcup_{i} D_{i} \cup \bigcup_{j} b_{j}$ is orientable. We will see that any $n$-tangle has a bicollared disk-band representation (Proposition 5).

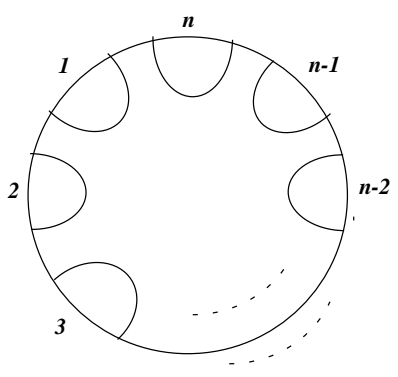

Fig. 1

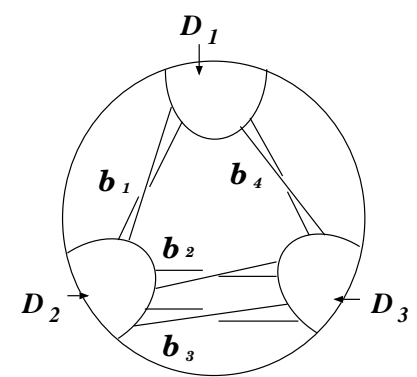

Fig. 2

A framed link is a disjoint union of embedded annuli in a 3-manifold. Framed links in $S^{3}$ can be identified with links whose each component is assigned an integer. Such links are also called framed links. Let $M$ be a three-manifold and $\mathcal{L}$ a framed link in $M$. We denote by $\Sigma(\mathcal{L}, M)$ the manifold that is obtained from $M$ by the surgery along $\mathcal{L}[\mathbb{8}$.

The case of two-fold branched covers is easy to visualize so we formulate it first.

Theorem 1. Let $\Omega\left(T_{0} ;\left\{D_{1}, \cdots, D_{n}\right\},\left\{b_{1}, \cdots, b_{m}\right\}\right)$ be a disk-band representation of an $n$-tangle $T$ in $B^{3}$. Let $\varphi: H_{0} \rightarrow B^{3}$ be the two-fold branched cover of $B^{3}$ by a genus $n-1$ handlebody $H_{0}$ branched along $T_{0}$. Then the two-fold branched cover of $B^{3}$ branched along $T$ has a surgery description $\Sigma\left(\varphi^{-1}\left(\bigcup_{i} b_{i}\right), H_{0}\right)$ (compare Fig. 3).

\footnotetext{
${ }^{1}$ Tangles are considered up to ambient isotopy but in practice we will often use a tangle for a tangle diagram or an actual embedding of a one-manifold.

${ }^{2}$ It contains information on the tangle, its surface and the decomposition of the surface.
} 

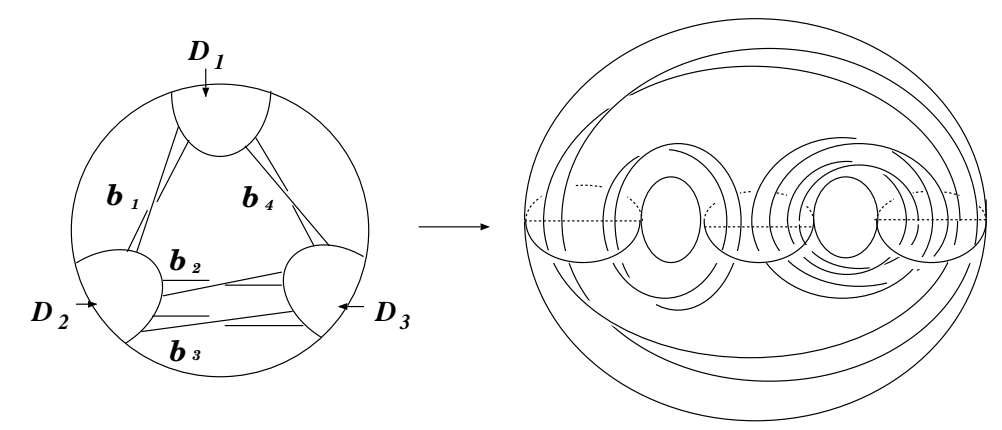

$\Omega\left(\boldsymbol{T}_{0} ;\left\{\boldsymbol{D}_{1,}, \boldsymbol{D}_{2,} \boldsymbol{D}_{3}\right\},\left\{\boldsymbol{b}_{1}, \boldsymbol{b}_{3}, \boldsymbol{b}_{3}, \boldsymbol{b}_{4}\right\}\right)$

$\Sigma\left(\varphi^{-1}\left(\mathbf{U}_{i} \boldsymbol{b}_{i}\right), \boldsymbol{H}_{\boldsymbol{o}}\right)$

Fig. 3

Proof. Let $X$ be $B^{3}-\bigcup_{i} D_{i}$ compactified with two copies, $D_{i}^{ \pm}$, of $D_{i}(i=1,2, \cdots, n)$ (Fig. 4). Let $X_{1}$ and $X_{2}$ be two copies of $X$, and let $D_{i, k}^{ \pm} \subset X_{k}$ denote copies of $D_{i}^{ \pm}$(i= $1,2, \cdots, n, k=1,2)$ (Fig. 5). Then $H_{0}$ is obtained from $X_{1} \cup X_{2}$ by identifying $D_{i, 1}^{\varepsilon}$ with $D_{i, 2}^{-\varepsilon}(\varepsilon \in\{-,+\})$. Let $b_{j, k}=\varphi^{-1}\left(b_{j}\right) \cap X_{k}$ and let $Y$ be $H_{0}-\bigcup_{j, k} b_{j, k}$ compactified with two copies $b_{j, k}^{ \pm}$of $b_{j, k}$ in $X_{k}(j=1,2, \cdots, m, k=1,2)$. Here, + or - sides of $D_{i, k}$ and $b_{j, k}$ are not necessarily compatible. We note, and it is the key observation of the construction, that the two-fold branched cover $H$ of $B^{3}$ branched along $T$ is obtained from $Y$ by identifying $b_{j, 1}^{\varepsilon}$ with $b_{j, 2}^{-\varepsilon}(\varepsilon \in\{-,+\})$. Note that each $b_{j, 1}^{+} \cup b_{j, 2}^{-} \cup b_{j, 1}^{-} \cup b_{j, 2}^{+}$is a torus. Let $c_{j}$ be the core of the annulus $b_{j, 1}^{+} \cup b_{j, 2}^{-}$. The manifold obtained from $Y$ by identifying $b_{j, 1}^{\varepsilon}$ with $b_{j, 2}^{-\varepsilon}$ is homeomorphic to the one obtained from $Y$ by attaching tori $D_{j}^{2} \times S^{1}(j=1,2, \ldots, m)$ so that $\partial D_{j}^{2}=c_{j}$. Hence $H$ is homeomorphic to the manifold with the surgery description $\Sigma\left(\varphi^{-1}\left(\bigcup_{j} b_{j}\right), H_{0}\right)$.

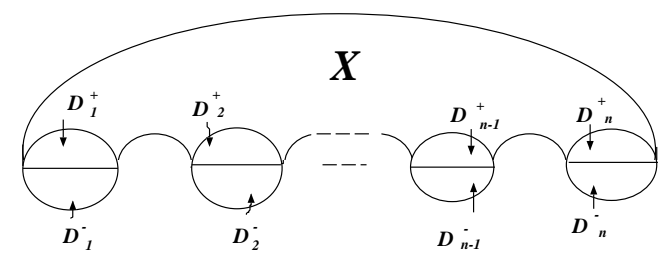

Fig. 4
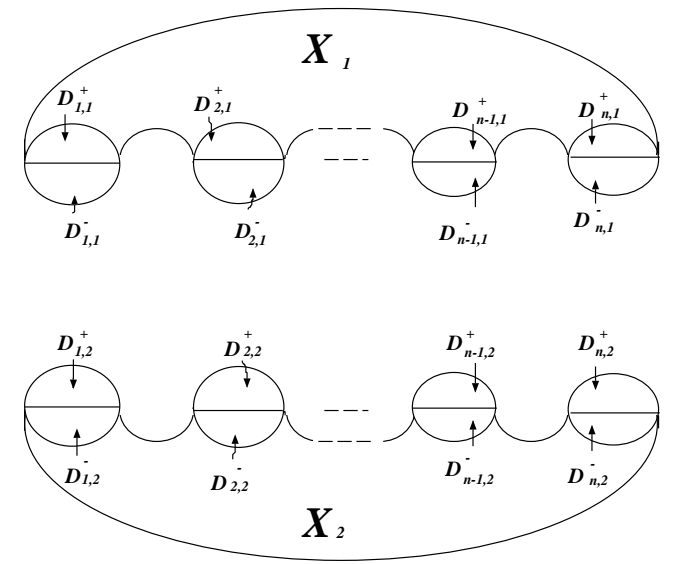

Fig. 5 
Example 2. (a) The two-fold branched cover $M^{(2)}\left(T_{1}\right)$ branched along a tangle $T_{1}$ in Fig. 6 is the Seifert manifold with the base a disk and two special fibers of type $(2,1)$ and $(2,-1)$. Furthermore $M^{(2)}\left(T_{1}\right)$ is a twisted $I$-bundle over the Klein bottle (for example see [6]). In particular, $\pi_{1}\left(M^{(2)}\left(T_{1}\right)\right)=\left\langle a, b \mid a b a^{-1} b=1\right\rangle$.

(b) If we glue together two copies of $T_{1}$ as in Fig. 7, we get a Borromean rings $L$. Thus our previous computation shows that the two-fold branched cover $M^{(2)}(L)$ of $S^{3}$ branched along $L$ is a "switched" double of the twisted $I$-bundle over the Klein bottle (see Fig. 8 for a surgery description). The fundamental group $\pi_{1}\left(M^{(2)}(L)\right)=\left\langle x, a \mid x^{2} a x^{2} a^{-1}, a^{2} x a^{2} x^{-1}\right\rangle$ is a 3-manifold group which is torsion free but not left orderable [9].

(c) If we take the double of the tangle $T_{1}$, we obtain the link in Fig. 9. The two-fold branched cover of $S^{3}$ branched along this link is the double of twisted $I$-bundle over Klein bottle. A surgery description of this manifold is shown in Fig. 10. Thus this manifold is the Seifert manifold of type $(2,1),(2,1),(2,-1),(2,-1)$. This manifold also has another Seifert fibration, which is a circle bundle over the Klein bottle.

Example 2(a) was motivated by the fact that the tangle $T_{1}$ yields a virtual Lagrangian of index 2 in the symplectic space of the Fox Z-colorings of the boundary of our tangle [5].

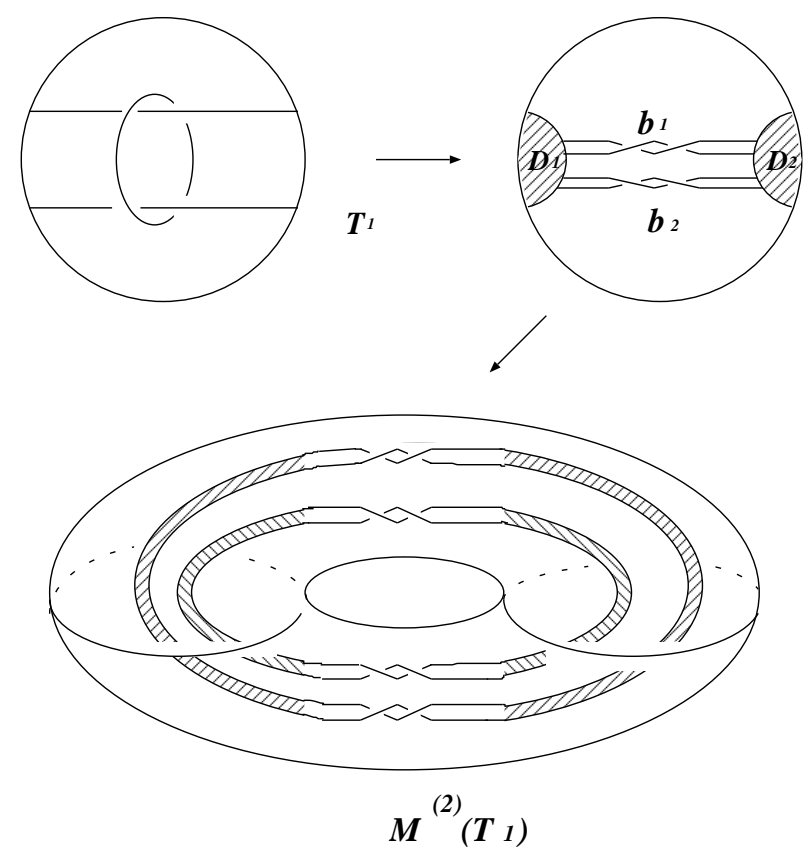

Fig. 6 


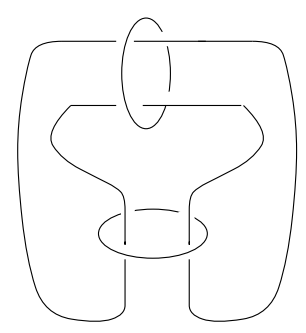

Fig. 7

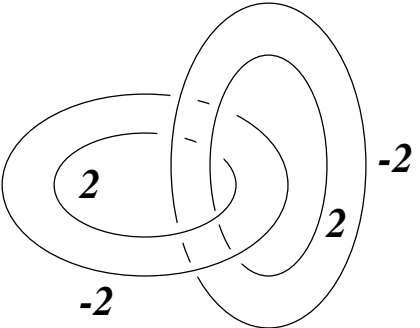

Fig. 8

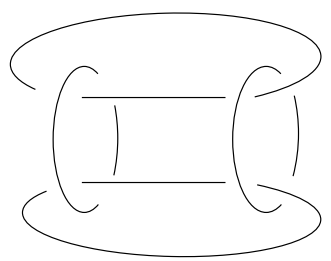

Fig. 9

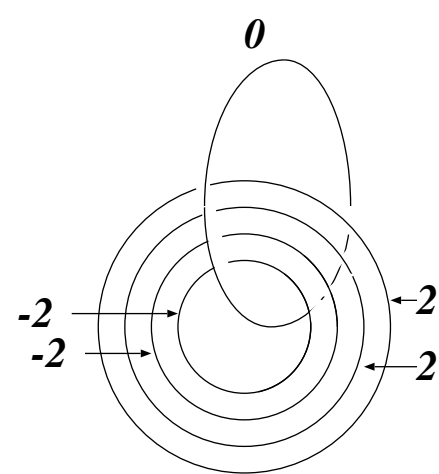

Fig. 10

More generally we have:

Example 3. Consider a tangle $T_{2}$ in Fig. 11, called a pretzel tangle of type $\left(a_{1}, a_{2}, \cdots, a_{n}\right)$, where each $a_{i}$ is an integer indicating the number of half-twists $(i=1,2, \cdots, n)$. The twofold branched cover $M^{(2)}\left(T_{2}\right)$ branched along the tangle $T_{2}$ is a Seifert fibered manifold with the base a disk and $n$ special fibers of type $\left(a_{1}, 1\right),\left(a_{2}, 1\right), \cdots,\left(a_{n}, 1\right)$ (Fig. 12).

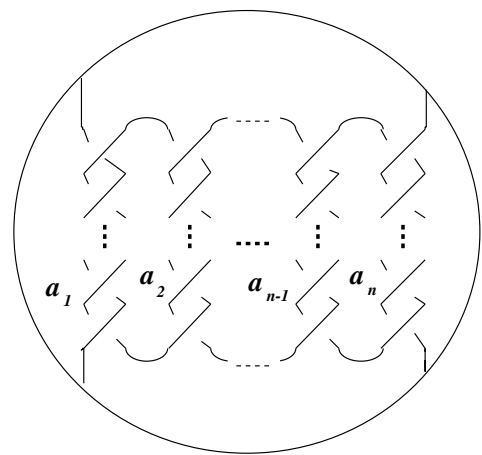

Fig. 11

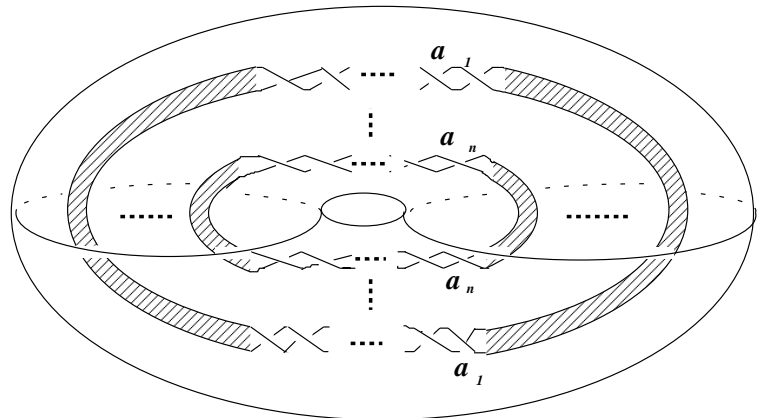

Fig. 12

Theorem 1 can be generalized to the $p$-fold cyclic branched cover assuming that a disk-band representation is bicollared. We proceed as follows:

Let $T=\Omega\left(T_{0} ;\left\{D_{1}, \cdots, D_{n}\right\},\left\{b_{1}, \cdots, b_{m}\right\}\right)$ be a bicollared disk-band representation of an $n$-tangle. Then $\bigcup_{i} D_{i} \cup \bigcup_{j} b_{j}$ has bicollar neighbourhood $\left(\cup_{i} D_{i} \cup \bigcup_{j} b_{j}\right) \times[-1,1]$. Let $X=\overline{B^{3}-\left(\left(\bigcup_{i} D_{i}\right) \times[-1,1]\right)}$ and $D_{i}{ }^{ \pm}=\left(D_{i} \times[ \pm 1,0]\right) \cap \partial X$. Let $X_{k}$ be a copy of $X$ and $D_{i, k}^{ \pm} \subset \partial X_{k}$ a copy of $D_{i}^{ \pm}(k=1,2, \cdots, p)$. Then the $p$-fold cyclic branched cover $\varphi: H_{0} \rightarrow B^{3}$ branched along $T_{0}$ is obtained from $X_{1} \cup \cdots \cup X_{p}$ by identifying $D_{i, k}^{+}$with $D_{i, k+1}^{-}(k=1, \cdots, p)$, where $k$ is considered modulo $p$. Let $b_{j, k}^{ \pm}=\varphi^{-1}\left(b_{j} \times\{ \pm 1\}\right) \cap X_{k}$. 
Note that each $b_{j, k}^{+} \cup b_{j, k+1}^{-}$is an annulus in $H_{0}$ for any $j$ and $k$. Then we obtain the $p$-fold cyclic branched cover of $B^{3}$ branched along $T$ in the similar way as in Theorem 1.

Theorem 4. Let $\Omega\left(T_{0} ;\left\{D_{1}, \cdots, D_{n}\right\},\left\{b_{1}, \cdots, b_{m}\right\}\right)$ be a bicollared disk-band representation of an $n$-tangle $T$ in $B^{3}$. Then $\Sigma\left(\bigcup_{j=1}^{m}\left(\bigcup_{k=1}^{p-1}\left(b_{j, k}^{+} \cup b_{j, k+1}^{-}\right)\right), H_{0}\right)$ is the p-fold cyclic branched cover of $B^{3}$ branched along $T$.

Note that we do not use the annuli $b_{j, p+1}^{+} \cup b_{j, 1}^{-}(j=1,2, \cdots, m)$ in the theorem above. In fact the cores of these annuli bound mutually disjoint 2-dsks in $\Sigma\left(\bigcup_{j=1}^{m}\left(\bigcup_{k=1}^{p-1}\left(b_{j, k}^{+} \cup\right.\right.\right.$ $\left.\left.\left.b_{j, k+1}^{-}\right)\right), H_{0}\right)$.

Proof. Let $Y=\overline{H_{0}-\bigcup_{j} \varphi^{-1}\left(b_{j} \times[-1,1]\right)}, V_{j, k}^{ \pm}=\varphi^{-1}\left(b_{j} \times[ \pm 1,0]\right) \cap X_{k}$ and $\beta_{j, k}^{ \pm}=$ $V_{j, k}^{ \pm} \cap Y\left(=\partial V_{j, k}^{ \pm} \cap \partial Y\right)$. Note that $\bigcup_{j} \varphi^{-1}\left(b_{j} \times[-1,1]\right)$ is a genus $p-1$ handlebody. Then the $p$-fold cyclic branched cover of $B^{3}$ branched along $T$ is homeomorphic to a manifold $H$ that is obtained from $Y$ by identifying $\beta_{j, k}^{+}$with $\beta_{j, k+1}^{-}(k=1, \cdots, p)$, where $k$ is taken modulo p. Moreover $H$ is homeomorphic to a manifold obtained from $\overline{H_{0}-\bigcup_{j} \varphi^{-1}\left(b_{j} \times[-1,1]\right)} \cup$ $\bigcup_{j}\left(V_{j, 1}^{-} \cup V_{j, p}^{+} \cup \varphi^{-1}\left(b_{j} \times\{0\}\right)\right)$ by identifying $\beta_{j, k}^{+}$and $b_{j, k}$ with $\beta_{j, k+1}^{-}$and $b_{j, k+1}(j=$ $1, \cdots, m, k=1, \cdots, p-1)$ respectively, where $b_{j, k}=\varphi^{-1}\left(b_{j} \times\{0\}\right) \cap X_{k}$. Note that $\beta_{j, k}^{+} \cup b_{j, k} \cup \beta_{j, k+1}^{-} \cup b_{j, k+1}$ is a torus. By the argument similar to that in the proof of Theorem 1, we have the required result.

Example 5. The three-fold cyclic branched cover $M^{(3)}\left(4_{1}\right)$ of $S^{3}$ branched along the figure eight knot has a surgery description shown in Fig. 13(a). The framed link in Fig. 13(a) can be deformed into the link in Fig. 13(b) by an ambient isotopy and a second Kirby move. The link in Fig 13(b) is ambient isotopic to the link in Fig 8. Hence $M^{(3)}\left(4_{1}\right)$ is homeomorphic to the two-fold branched cover of $S^{3}$ branched along the Borromean rings (cf. Example 2(b)).

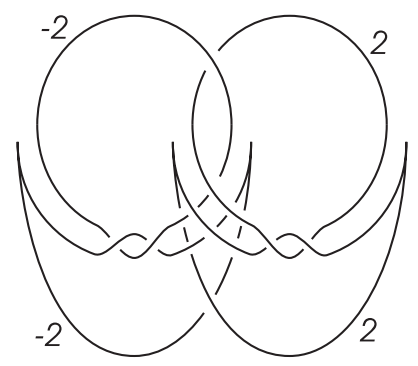

(a)

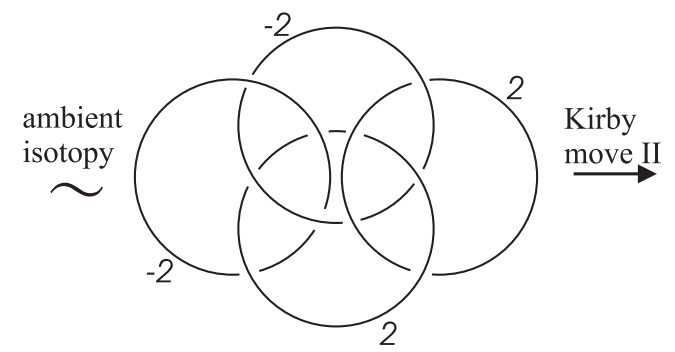

Fig. 13

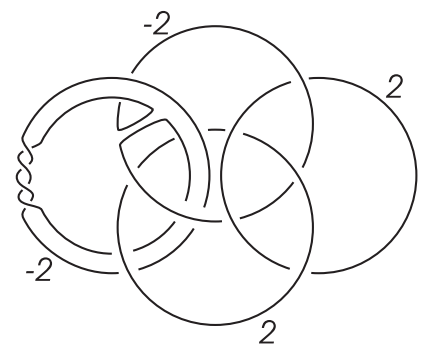

(b)

Proposition 6. Any n-tangle $\left(B^{3}, T\right)$ has a (bicollared) disk-band representation. 
Proof. We attach a trivial $n$-tangle $T_{0}$ to the $n$-tangle $T$ by a homeomorphism $\varphi$ : $\partial\left(B, T_{0}\right) \rightarrow \partial(B, T)$. We obtain a link $L=T_{0} \cup T$ in a three-sphere $B \cup_{\varphi} B$ with a diagram $D\left(T_{0} \cup T\right)$ as in Fig. 14. We may assume that the diagram $D\left(T_{0} \cup T\right)$ is connected. We color, in checkerboard fasion, the regions of the plane cut by the diagram $D\left(T_{0} \cup T\right)$ and chose $n$ points $\left\{v_{1}, v_{2}, \cdots, v_{n}\right\}$ as in Fig. 15. Since $D\left(T_{0} \cup T\right)$ is connected, there is a spine $G$ of the black surface with the vertex $\operatorname{set}\left\{v_{1}, v_{2}, \cdots, v_{n}\right\}$. By retracting the black regions into the neighbourhood of $G$ and restricting to $B^{3}$, we have a required surface.

When we use the Seifert algorithm instead of checkerboard coloring, we always obtain a bicollared disk-band representation.

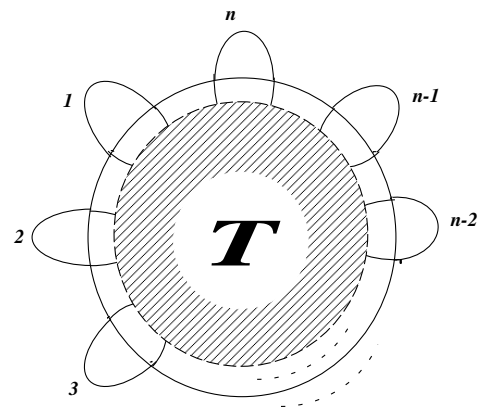

Fig. 14

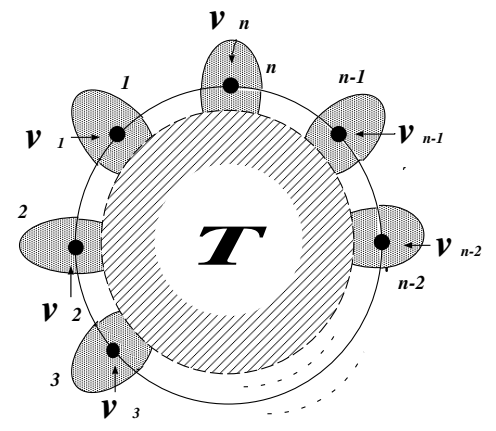

Fig. 15

\section{Heegaard decompositions}

In addition to the surgery presentation, it is also useful to have another presentation of a $p$-fold cyclic branched cover. Our construction leads straightforwardly to a Heegaard decomposition, a decomposition into a compression body [2, 3] and a handlebody, of a $p$-fold cyclic branched cover.

Let $F$ be a connected surface in $B^{3}$ bounded by an $n$-tangle $T$ and $n$ arcs in $\partial B^{3}$. The surface $F$ is defined to be free if the exterior of $F$ is homeomorphic to $\left(S_{n} \times I\right) \cup(1$ handles), where $S_{n}$ is an $n$-punctured sphere and the all attaching points of the 1-handles are contained in $S_{n} \times\{0\}$. As we observed before, any connected surface has disk-band decomposition. A disk-band representation $\Omega\left(T_{0} ;\left\{D_{1}, \cdots, D_{n}\right\},\left\{b_{1}, \cdots, b_{m}\right\}\right)$ is defined to be free if the surface $\bigcup_{i} D_{i} \cup \bigcup_{j} b_{j}$ is free.

First we consider the case of two-fold branched covers. The following algorithm gives a Heegaard decomposition of $M^{(2)}(T)$. Start from a free disk-band representation of $T$. Then we have a surgery description $\Sigma\left(\varphi^{-1}\left(\bigcup_{i} b_{i}\right), H_{0}\right)$ of $M^{(2)}(T)$ (Fig. 3). Let $\alpha_{1}, \alpha_{2}, \ldots, \alpha_{2 m-n}$ be the $2 m-n$ arcs which are the connected components of $T_{0}-\bigcup_{j} b_{j}$ 
contained in the interior of $B^{3}$. Then the complement of $\varphi^{-1}\left(\bigcup_{i} b_{i} \bigcup_{l} \alpha_{l}\right)$ and the arcs is a compression body. Then $M^{(2)}(T)$ is obtained from the compression body by gluing the handle body as follows; (i) adding meridian disks of the arcs, and (ii) filling the rest according to the surgery description. For the tangle $T_{1}$ in Example 2, a Heegaard decomposition of $M^{(2)}\left(T_{1}\right)$ is given as in Fig. 16.

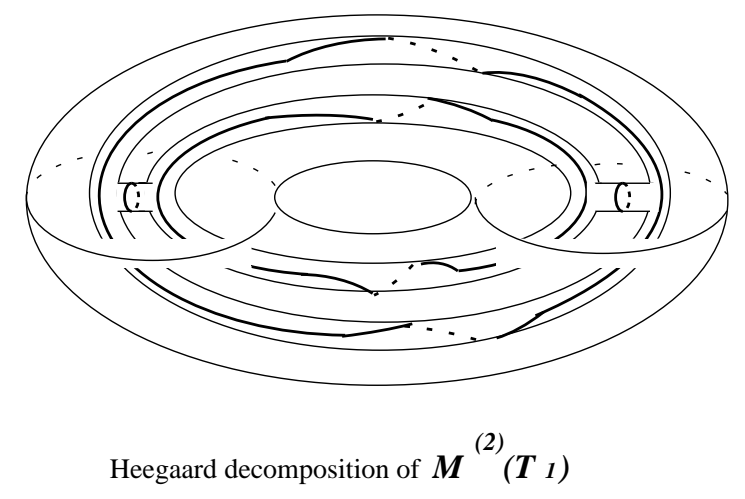

Fig. 16

The similar method gives a Heegaard decomposition of a $p$-fold cyclic branched cover. Our construction is a modification of the construction in the proof of Theorem 4. The handlebody part of the decomposition is obtained from the handlebodies $\cup_{j} \varphi^{-1}\left(b_{j} \times\right.$ $[-1,1])$ by connecting them using $2 m-n$ "tubes" along $\varphi^{-1}\left(T_{0}\right)$. We get genus $m p-n+1$ handlebody.

From the observation above it follows that:

Theorem 7. Let $\Omega\left(T_{0} ;\left\{D_{1}, \cdots, D_{n}\right\},\left\{b_{1}, \cdots, b_{m}\right\}\right)$ be a free, bicollared disk-band representation of an n-tangle $T$ in $B^{3}$. Let $\varphi: H_{0} \rightarrow B^{3}$ be the $p$-fold cyclic branched cover of branched along $T_{0}$. Let $\alpha_{1}, \alpha_{2}, \ldots, \alpha_{2 m-n}$ be the $2 m-n$ arcs which are the connected components of $T_{0}-\cup_{j} b_{j}$ contained in the interior of $B^{3}$. Then the following holds.

(a) The complement $W=\overline{H_{0}-\varphi^{-1}\left(\bigcup_{j} b_{j} \times[-1,1] \cup \bigcup_{l} N\left(\alpha_{l}\right)\right)}$ is a compression body, where $N\left(\alpha_{l}\right)$ is the tubular neighbourhood of $\alpha_{l}$ in $B^{3}$.

(b) The p-fold cyclic branched cover of $B^{3}$ branched along $T$ has a Heegaard decomposition into the compression body $W$ and a genus $m p-n+1$ handlebody.

(c) The gluing map is given by the curves $c_{j, k}(j=1,2, \ldots, m, k=1,2, \ldots, p-1)$ and $m_{l}(l=1,2, \ldots, 2 m-n)$ in $\partial W$, where $c_{j, k}$ is the core of the annulus $b_{j, k}^{+} \cup b_{j, k+1}^{-}$in Theorem 4 and $m_{l}$ is the meridian curve of $\varphi^{-1}\left(N\left(\alpha_{l}\right)\right)$.

In the theorem above, the assumption that a disk-band representation is bicollared is not necessary in the case that $p=2$. The curves $c_{j, k}(j=1,2, \ldots, m, k=1,2, \ldots, p-1), m_{l}(l=$ 
$1,2, \ldots, 2 m-n)$ are essential, $m p-n+1$ of them are nonseparating and $m-1$ curves, $m_{l}$ 's, are separating.

Remark 8. Since the surfaces given in the proof of Proposition 5 are connected and free, we can use them to find Heegaard decompositions of branched cyclic covers. Let $c$ denote the crossing number of a connected diagram $D\left(T_{0} \cup T\right), b$ the number of the black regions and $s$ the number of the Seifert circles of $D\left(T_{0} \cup T\right)$. Then we have a Heegaard decomposition of $M^{(2)}(T)$ (resp. $M^{(p)}(T)$ ) of the genus $n+2 c-2 b+1$ (resp. $p(n+c-s)-n+1)$.

\section{References}

[1] S. Akbulut and R. Kirby: Branched covers of surfaces in 4-manifolds, Math. Ann. 252 (1980), 111-131.

[2] F. Bonahon and J. P. Otal: Scindements de Heegaard des espaces lenticulaires, Ann. Sci. Ec. Norm. Sup. 16 (1983), 451-466.

[3] A. J. Casson and C. McA. Gordon: Reducing Heegaard splittings, Topology. Appl. 27 (1987), 275-283.

[4] J. H. Conway: An enumeration of knots and links and some of their related properties, Computational problems in Abstract Algebra, Proc. Conf. Oxford 1967, Pergamon Press (1970), 329-358.

[5] J. Dymara, T. Januszkiewicz, J. H. Przytycki: Symplectic structure on Colorings, Lagrangian tangles and Tits buildings, preprint (May 2001).

[6] W. Jaco: Lectures on three manifold topology, Conference board of Math. 43, A.M.S. (1980).

[7] J. M. Montesinos: Surgery on links and double branched covers of $S^{3}$, Knots, groups and 3-manifolds, Ann. Math. Studies, Princeton Univ. Press. 84 (1975), 227-259.

[8] V. V. Prasolov and A. B. Sossinsky: Knots, links, braids and 3-manifolds, A.M.S. (1997).

[9] D. Rolfsen: Maps between 3-manifolds with nonzero degree: a new obstruction, in preparation for Proceedings of New Techniques in Topological Quantum Field Theory, NATO Advanced Research Workshop, August 2001, Canada. 